\title{
Ultrafast laser-induced dynamics of noncollinear spin structures in amorphous NdFeCo and PrFeCo
}

\author{
J. Becker, ${ }^{1,2}$ A. Tsukamoto, ${ }^{3}$ A. Kirilyuk,,${ }^{1}$ J. C. Maan, ${ }^{2}$ Th. Rasing, ${ }^{1}$ P. C. M. Christianen, ${ }^{2}$ and A. V. Kimel ${ }^{1}$ \\ ${ }^{1}$ Radboud University, Institute for Molecules and Materials, Nijmegen 6525 AJ, The Netherlands \\ ${ }^{2}$ High Field Magnet Laboratory (HFML - EMFL), Radboud University, 6525 ED Nijmegen, The Netherlands \\ ${ }^{3}$ College of Science and Technology, Nihon University, 7-24-1 Funabashi, Chiba, Japan
}

(Received 28 May 2015; revised manuscript received 23 October 2015; published 9 November 2015)

\begin{abstract}
The fanned out, noncollinear spin structure of the $\mathrm{Fe}(\mathrm{Co})$ sublattice in amorphous $\mathrm{NdFeCo}$ and PrFeCo alloys is shown to strongly affect its ultrafast laser-induced magnetization dynamics. An overshooting effect is discovered at low applied magnetic fields, where the magnetization temporarily increases above its equilibrium value. We explain this phenomenon by considering the dynamics of the noncollinear spin structure. After femtosecond laser excitation the system first reconstructs magnetic order on a time scale of 50-150 ps arriving at a state with a smaller opening angle of the fan. Subsequently, the original opening angle is restored on a time scale of nanoseconds. Increasing the field up to $0.6 \mathrm{~T}$ we can fully close the fan and therewith suppress the overshooting behavior.
\end{abstract}

DOI: 10.1103/PhysRevB.92.180407

Magnetic structures in materials come in much greater variety than the basic collinear ferro- and antiferromagnetic ordering. There is an intriguing diversity of noncollinear spin structures ranging from completely disordered spin glass states to canted structures in frustrated systems and even more exotic constructs, such as magnetic vortices and skyrmions [1,2]. In the past decade, research directed towards modeling and understanding these structures has intensified as they became increasingly interesting within the rising field of spintronics. Understanding and controlling the magnetic degrees of freedom and especially the dynamics of these noncollinear structures are thought to be key elements in developing next generation memory devices [3].

Amorphous magnets such as amorphous rare earthtransition metal (RE-TM) alloys are one class of materials which is known to show noncollinear magnetic behavior [4]. After RE-TM alloys were recognized in the 1970s for their unique, easily tunable magnetic properties and used as a reliable magneto-optical storage medium, they moved again, recently, into the focus of the scientific community with the demonstration of all optical magnetic switching (AOS) in GdFeCo [5]. Magnetic order in RE-TM alloys is built up by two coupled magnetic sublattices consisting of the RE and TM magnetic moments. In most of the RE-TM alloys, the alignment of these moments is not collinear, but sperimagnetic in the sense of Coey et al. [4]. In this sperimagnetic structure, one or both of the magnetic sublattices show a fanlike distribution of the magnetic moments around a preferred direction of magnetization, which is caused by competing exchange interactions and single-ion anisotropy at the respective sites of the TM or RE atoms. The interplay between these competing magnetic interactions could possibly lead to yet unexplored nonequilibrium magnetic phases on a ps time scale. So far, however, little is known about the effect of this noncollinear magnetic structure on the spin dynamics as most studies focus on longitudinal and precessional dynamics [6-8].

In this Rapid Communication we report static characterization and time-resolved magneto-optical studies of ultrafast laser-induced magnetization dynamics in the light RE-TM alloys $\mathrm{NdFeCo}$ and PrFeCo. Our results show that the TM sublattice in both alloys indeed possesses a noncollinear
PACS number(s): 75.40.Gb, 42.62.Fi, 75.40.Cx, 75.50.Kj

magnetic structure, which leads to a sudden increase in magnetization above its equilibrium value in response to a femtosecond laser excitation. This unexpected behavior of the magnetization dynamics can be described by a transient ferromagnetic alignment of the TM moments.

The investigated samples are prepared using magnetron sputtering and have the same general multilayer structure. A $20 \mathrm{~nm}$ RE-TM amorphous film is deposited on a glass |AlTi(10 nm) |SiN(5 nm) stack. The AlTi film serves as a heat sink, whereas the dielectric $\mathrm{SiN}$ is used as the buffer layer. The samples are capped by $60 \mathrm{~nm} \mathrm{SiN}$ to prevent oxidation while also being highly transparent in the visible to near infrared, allowing for optical access to the RE-TM layer. The RE-TM layer is of the form $\mathrm{RE}_{x} \mathrm{Fe}_{100-(x+y)} \mathrm{Co}_{y}$. In this Rapid Communication, we report results on two representative samples with different light RE elements, $\mathrm{Nd}_{30} \mathrm{Fe}_{61.3} \mathrm{Co}_{8.7}$ and $\mathrm{Pr}_{20} \mathrm{Fe}_{70} \mathrm{Co}_{10}$ (hereafter referred to as Nd$\mathrm{FeCo}$ and $\mathrm{PrFeCo}$ ). For comparison, two samples containing the heavy RE elements gadolinium and dysprosium were also investigated. We used conventional static and time-resolved (TR) magneto-optical Kerr effect (MOKE) methods [9] to study the static and dynamic magnetic properties of these samples.

Figure 1(a) shows typical MOKE hysteresis loops at room temperature of the two light RE-TM samples. The samples were probed with light of $630 \mathrm{~nm}$ central wavelength corresponding to a photon energy of $1.97 \mathrm{eV}$. In principle, this photon energy is not sufficient to excite the transitions involving $4 f$ states, which carry most of the RE magnetic moment and lie at energies higher than $3 \mathrm{eV}[10,11]$. Even though photoemission spectra show splitting of the $4 f$ peaks in certain Pr and Nd-TM alloys [12] to energies below $2 \mathrm{eV}$, the magneto-optical spectra of our samples between 1 and $3 \mathrm{eV}$ are very similar to those of pure $\mathrm{Fe}$ (see Supplemental Material [13]). Therefore we believe our measurements to be mainly sensitive to the TM sublattice but cannot completely rule out a RE contribution to the signal.

The external magnetic field $\mu_{0} \mathbf{H}$ was applied along the in-plane easy axis of magnetization [inset Fig. 1(a)]. The results for the PrFeCo [Fig. 1(a) top] and the NdFeCo [Fig. 1(a) bottom] sample show specific similarities. The loops are 


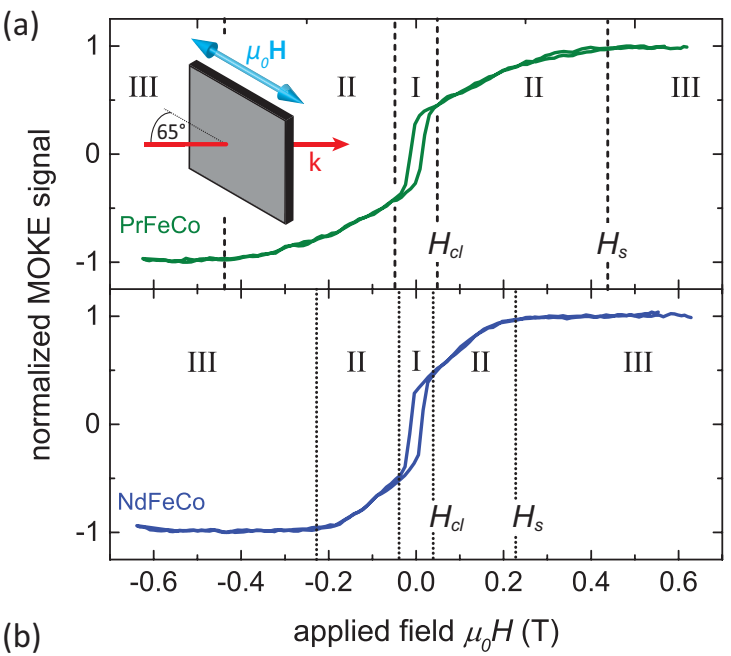

(b)

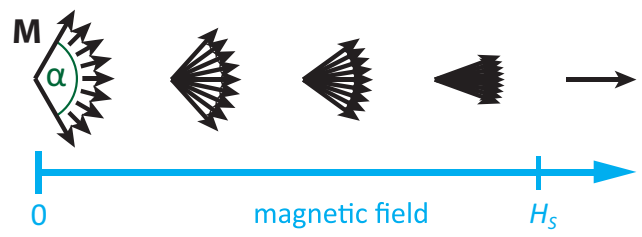

FIG. 1. (Color online) (a) MOKE hysteresis loops of PrFeCo and $\mathrm{NdFeCo}$ at room temperature and schematic of the experimental geometry (inset). A paramagnetic background was subtracted from the measurements. Vertical lines indicate the boundaries of regions I, II, and III. (b) Schematic representation of the closing of a magnetic fan structure with opening angle $\alpha$.

composed of three distinct regions, labeled I, II, and III. Region I is the "main" hysteresis loop wherein the magnetization switches and thus determines the coercive field. The loop closes at a certain field $H_{\mathrm{cl}}$. At fields larger than $H_{\mathrm{cl}}$ the signal continuously rises in region II until it reaches saturation at $H_{\mathrm{S}}$, which defines the onset of region III. This onset occurs at $\sim 0.44 \mathrm{~T}$ for PrFeCo and $\sim 0.23 \mathrm{~T}$ for $\mathrm{NdFeCo}$. Magnetization curves with the field applied perpendicularly to the film plane show no hysteresis loop but only linear behavior (see Supplemental Material [13]).

To understand these results, one has to take into account the sperimagnetic structure of the samples, implying a fanlike distribution of the magnetic moments in possibly both magnetic sublattices. For the TM sublattice, to which our measurements are most sensitive, the TM-TM exchange $J_{\mathrm{TM}-\mathrm{TM}}$ is dominating over the RE-TM exchange $J_{\mathrm{RE}-\mathrm{TM}}$ and local anisotropy. In RE-Fe compounds, experiments [14-19] backed up by theoretical models [20-22] suggest that the Fe sublattice has a noncollinear, fanlike magnetic structure with opening angle $\alpha$ [Fig. 1(b)] caused by competing ferromagnetic and antiferromagnetic exchange interactions. This magnetic frustration arises due to nonuniform distances between neighboring Fe atoms. For the RE sublattice on the other hand, it was shown that the strong random single-ion anisotropy at the RE sites will lead to a distribution of magnetic moments around an easy axis [14,23,24]. The opening angle of this fan structure depends on the relative strengths of $J_{\mathrm{RE}-\mathrm{TM}}$ and $J_{\mathrm{RE}-\mathrm{RE}}$ with respect to the single-ion anisotropy.
So in the NdFeCo and PrFeCo sample, both sublattices most probably have a noncollinear magnetic structure. Although we are measuring mainly the TM sublattice, the RE sublattice acts on the former through $J_{\mathrm{RE}-\mathrm{TM}}$.

The increasing magnetization in region II can then be explained with a transition from a noncollinear structure to a collinear ferromagnetic structure as the $\mathrm{Fe}(\mathrm{Co})$ magnetic moments align with increasing external field [Fig. 1(b)]. This effectively closes the fan as the Zeeman-interaction overcomes the effect of local magnetic frustration. In region III the loop is completely closed and the net sample magnetization saturated. This behavior has been observed in light RE-based alloys but was ascribed to the sperimagnetic structure of the RE element $[14,25,26]$. However, as in this experiment we probe the magnetization of the TM sublattice [10,11], the above described effect is evidence for a noncollinear magnetic structure in the $\mathrm{Fe}(\mathrm{Co})$ sublattice.

Assuming that the TM sublattice magnetization in region III is indeed collinear and thus equal to the saturation magnetization $M_{\mathrm{S}}$, one can extract the opening angle $\alpha$ of the fanlike structure in region II from the relative magnetization values in Fig. 1(a). The following formula gives the relation between the effective magnetization $M_{\text {eff }}$ and the opening angle $\alpha$ for the two-dimensional case of in-plane magnetization. It is derived from purely geometrical considerations and assumes a constant length (equal to $M_{\mathrm{S}}$ ) of the magnetization vectors, randomly distributed within an opening angle $\alpha$ :

$$
M_{\mathrm{eff}}=M_{\mathrm{S}} \frac{2}{\alpha} \int_{0}^{\alpha / 2} \cos \phi d \phi=M_{\mathrm{S}} \frac{2 \sin (\alpha / 2)}{\alpha} .
$$

Using Eq. (1) we can calculate the opening angles at the onset of region II to be $\alpha_{\mathrm{n}}=196^{\circ}$ for $\mathrm{NdFeCo}$ and $\alpha_{\mathrm{p}}=221^{\circ}$ for PrFeCo.

This behavior was not observed in DyFeCo and GdFeCo (see Supplemental Material [13]). As they are heavy RE-TM alloys, the RE-TM exchange coupling is negative, which was shown to facilitate collinear ferromagnetic alignment in the $\mathrm{Fe}(\mathrm{Co})$ sublattice [4]. Moreover, in the case of Gd, the RE-TM exchange is considerably stronger than for $\mathrm{Nd}$ and $\mathrm{Pr}$ and the Gd spin arrangement is collinear [4,14]. Even though there might be a fan structure, the closing effect might not be strong enough to show up in our measurements or might become visible only at even higher applied fields.

To investigate the influence of the noncollinear magnetic structure on the magnetization dynamics, region II to region III were studied in detail using TR-MOKE (Fig. 2), using the same experimental geometry as in the static MOKE experiment [inset Fig. 1(a)]. The material was both excited and probed with $100 \mathrm{fs}$ short laser pulses at a central wavelength of $800 \mathrm{~nm}$ for the pump and $630 \mathrm{~nm}$ for the probe. We again expect our measurements to be sensitive mostly to the magneto-optical response of the TM magnetic sublattice (see Supplemental Material [13]). All data shown here were extracted from measurements of both field polarities to eliminate nonmagnetic contributions to the signal. Figures 2(a) and 2(b) show the magnetization dynamics at a low pump fluence of $0.23 \mathrm{~mJ} / \mathrm{cm}^{2}$ for $\mathrm{PrFeCo}$ and $0.45 \mathrm{~mJ} / \mathrm{cm}^{2}$ for $\mathrm{NdFeCo}$. The probe fluence was $2 \mu \mathrm{J} / \mathrm{cm}^{2}$ for all measurements. In both samples an overshooting effect can clearly be observed at the lowest 


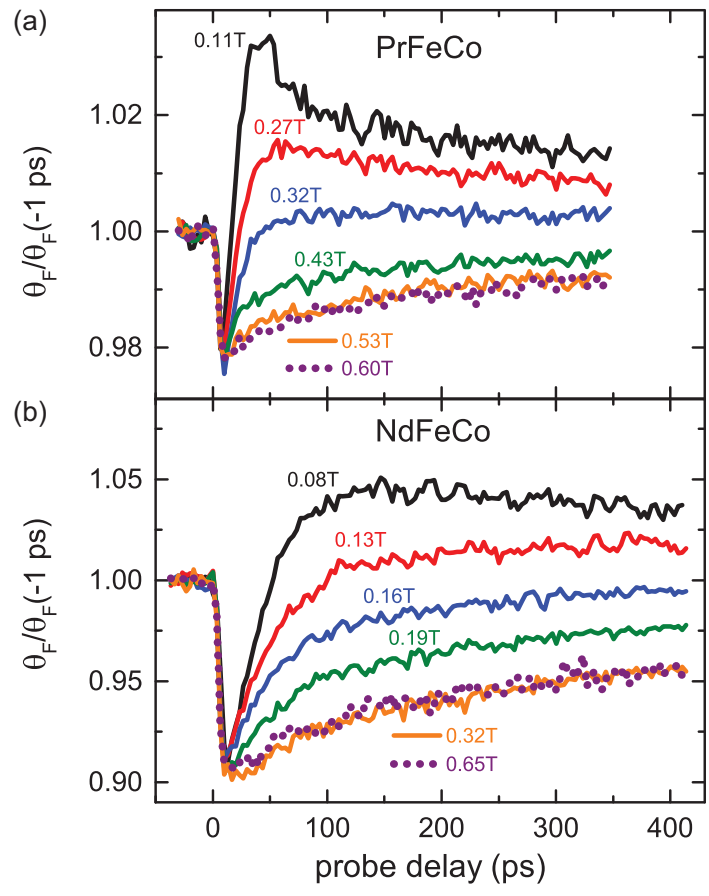

FIG. 2. (Color online) TR-MOKE data showing the time evolution of the Kerr rotation angle $\theta_{K}$ at different applied magnetic fields for $\mathrm{PrFeCo}$ (a) and $\mathrm{NdFeCo}$ (b) at room temperature. Overshooting behavior is clearly visible at low fields whereas the relaxation process does not change at the highest fields above $H_{\mathrm{S}}$.

applied magnetic fields: After an initial demagnetization, the signal increases to a value greater than at negative probe delay. This indicates a transient higher magnetization than in the unperturbed state before pump arrival. The effect is reduced with increasing magnetic field, showing a strongly field-dependent remagnetization, until it vanishes completely. Interestingly, a complete quenching of this field-dependent behavior occurs with the onset of region III (Fig. 2) for the respective sample. For fields above $H_{\mathrm{S}}$, the magnetization dynamics does not change. This field-dependent remagnetization and overshooting of the signal occurs exclusively in region II of the $\mathrm{NdFeCo}$ and $\mathrm{PrFeCo}$ samples and was not observed in $\mathrm{GdFeCo}$ and DyFeCo.

We here offer a phenomenological explanation by not only taking into account the longitudinal magnetization dynamics of the magnetic moments but also a dynamic response of the fan structure. If we assume that the pump stimulus causes the opening angle of the fan to first close, then relax back to its original equilibrium angle, we can retrace the observed overshooting behavior as a combination of the fan dynamics and longitudinal dynamics [Fig. 3(a)]. The following approach is used in order to single out and quantify the dynamics of the magnetic fan structure of the TM sublattice.

We first fit the remagnetization occurring in region III [blue curve, Fig. 3(a)] using a biexponential function, which is then subtracted from the measurements at fields in region II. Assuming that in a first approximation, the longitudinal dynamics of the magnetic moments-independently of the fan dynamicsdoes not change throughout region II and region III, this procedure reveals the fan dynamics only. As an example,
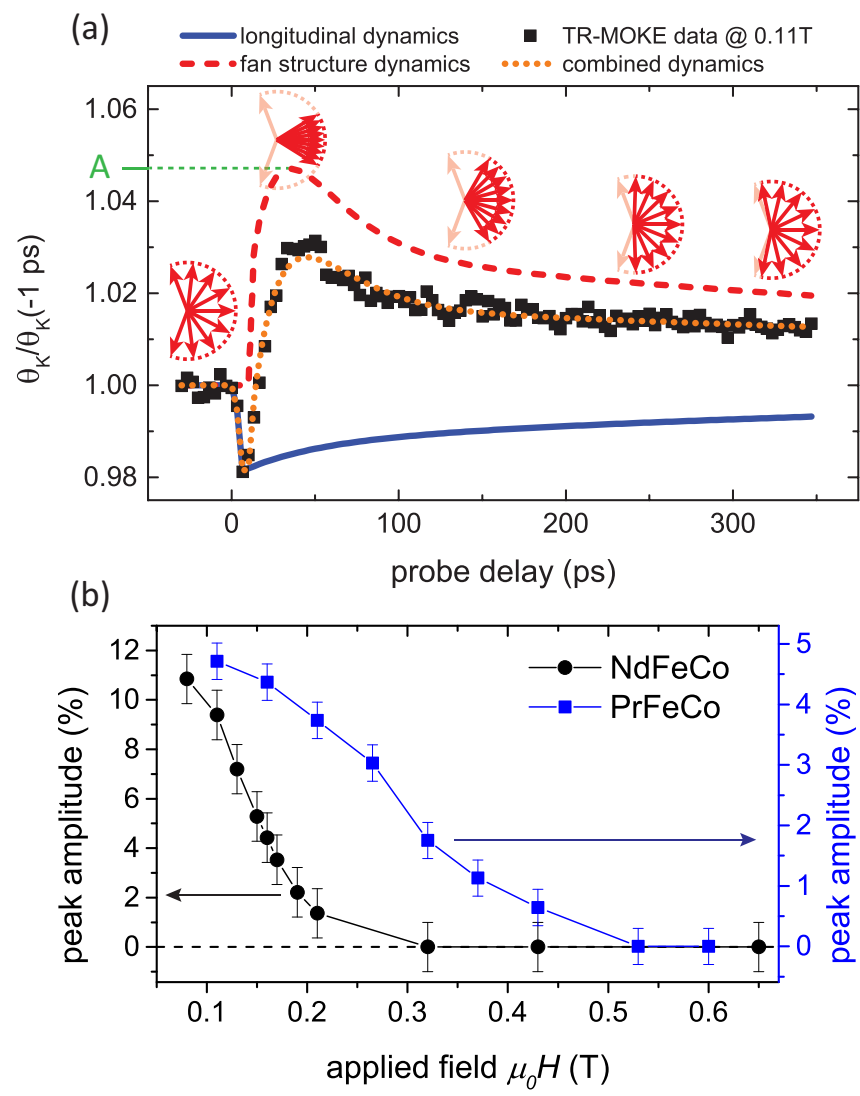

FIG. 3. (Color online) (a) Contributions from longitudinal dynamics (solid blue curve), fan structure dynamics (dashed red curve), and both dynamics combined (dotted orange curve) plotted together with TR-MOKE data at $0.11 \mathrm{~T}$ (black squares) for the PrFeCo sample at room temperature. The (green) A denotes the amplitude of the fan structure dynamics. The (red) arrows schematically show the evolution of the TM fan structure in one pump-probe cycle. (b) Peak amplitudes relative to $\theta_{F} / \theta_{F}(-1 \mathrm{ps})=1$ extracted from the fan dynamics [see dashed red curve in (a)] at different applied magnetic fields for the Nd- and PrFeCo sample.

Fig. 3(a) shows the separate and combined contributions of the fan (red dashes) and longitudinal dynamics (blue curve) for a low-field measurement on the PrFeCo sample. Using this procedure, one can extract the amplitude [green A in Fig. 3(a)] and delay position of the peak in the fan dynamics signal for all fields. The peak amplitudes shown in Fig. 3(b) clearly illustrate the disappearance of the overshooting effect towards higher applied fields. Furthermore, it is possible to deduce the change in the fan opening angle $\Delta \alpha$ using Eq. (1). It is assumed that the equilibrium opening angle before pump arrival for a measurement at a certain field corresponds to the value derived from the static measurements at the same field. For the lowest fields measured in region II we calculate a change in opening angle of $8^{\circ}$ at $0.11 \mathrm{~T}$ for $\mathrm{PrFeCo}$ and $20^{\circ}$ at $0.08 \mathrm{~T}$ for $\mathrm{NdFeCo}$.

The peak position, on the other hand, lies at a delay of $\sim 50$ ps for PrFeCo and $\sim 150$ ps for $\mathrm{NdFeCo}$ and changes little with field. However, first preliminary data suggest that it strongly depends on temperature (not shown) and pump fluence (see Supplemental Material [13]). A more rigorous study has yet to be performed. 
In the following, we give a tentative description of the mechanism leading to the overshooting phenomenon. Upon pump arrival, the electron system is heated up which leads to a partial demagnetization within the first couple of picoseconds. Electron-phonon interaction heats up the lattice until thermal equilibrium between electrons and lattice is reached. Taylor et al. [15], investigating sperimagnetic amorphous GdFeCo and GdFeNi alloys, showed evidence that increasing the temperature will in turn increase ferromagnetic alignment in the TM magnetic sublattice. This agrees with our measurement of magneto-optical hysteresis loops at different temperatures (see Supplemental Material [13]) which show a shrinking of region II upon increasing the temperature. As competing antiferromagnetic and ferromagnetic exchange interactions are the main force behind the fanning of the $\mathrm{Fe}(\mathrm{Co})$ magnetic moments, they are now (more) free to align collinearly. This effectively reduces the opening angle of the magnetization cone, leading temporarily to a higher net magnetization.

Depending on how fast this process occurs and on how much the longitudinal magnetization has recovered at that point, the measured signal can be above the initial value. Towards longer delay times the energy is dissipated and the local magnetic frustration, and with it the opening angle of the magnetic fan structure, recovers until it reaches its initial value. Following this mechanism one can explain the fielddependent behavior. The higher the applied field, the smaller the initial opening angle. Accordingly, the overshooting effect decreases along with the difference in net magnetization between the aligned state and the fan state and finally vanishes at $H_{\mathrm{S}}$.
In conclusion, we provided direct experimental evidence through static and dynamic magneto-optical measurements that the TM sublattice in Nd- and PrFeCo alloys possesses a fanlike magnetic structure. We showed that if stimulated by an ultrafast laser pulse, this fan structure undergoes its own dynamics, the results of which mix with the longitudinal dynamics of the magnetic moments. Upon applying an external magnetic field of sufficient strength to align all TM magnetic moments, the fan dynamics is suppressed and only the longitudinal response of the magnetic system remains. By increasing the magnetic field it is thus possible to switch between a regime determined by magnetic frustration to a regime purely governed by ferromagnetic exchange. This behavior might not only be limited to light RE-TM alloys but could potentially be a general feature of noncollinear spin structures.

The authors would like to thank I. Razdolski and T. J. Huisman for fruitful discussions and P. Albers, A. Toonen, and A. van Etteger for technical support. This research was partially supported by de Nederlandse Organisatie voor Wetenschappelijk Onderzoek (NWO), de Stichting voor Fundamenteel Onderzoek der Materie (FOM), the European Union's Seventh Framework Program (FP7/2007-2013) Grant No. 281043 (Femtospin), and the European Union's Seventh Framework Program (FP7/2007-2013)/ERC Grant Agreement No. 257280 (Femtomagnetism) and Grant Agreement No. 339813 (Exchange). Part of this work was also supported by EuroMagNET II under the EU Contract No. 228043. We acknowledge the support of the HFML-RU/FOM, member of the European Magnetic Field Laboratory (EMFL).
[1] J. M. D. Coey, Can. J. Phys. 65, 1210 (1987).

[2] N. Nagaosa and Y. Tokura, Nat. Nanotechnol. 8, 899 (2013).

[3] A. Fert, V. Cros, and J. Sampaio, Nat. Nanotechnol. 8, 152 (2013).

[4] J. M. D. Coey, J. Appl. Phys. 49, 1646 (1978).

[5] C. D. Stanciu, F. Hansteen, A. V. Kimel, A. Kirilyuk, A. Tsukamoto, A. Itoh, and T. Rasing, Phys. Rev. Lett. 99, 047601 (2007).

[6] M. van Kampen, C. Jozsa, J. T. Kohlhepp, P. LeClair, L. Lagae, W. J. M. de Jonge, and B. Koopmans, Phys. Rev. Lett. 88, 227201 (2002).

[7] C. D. Stanciu, A. V. Kimel, F. Hansteen, A. Tsukamoto, A. Itoh, A. Kiriliyuk, and T. Rasing, Phys. Rev. B 73, 220402(R) (2006).

[8] B. Koopmans, G. Malinowski, F. Dalla Longa, D. Steiauf, M. Fähnle, T. Roth, M. Cinchetti, and M. Aeschlimann, Nat. Mater. 9, 259 (2010).

[9] A. Kirilyuk, A. V. Kimel, and T. Rasing, Rev. Mod. Phys. 82, 2731 (2010).

[10] J. K. Lang, Y. Baer, and P. A. Cox, J. Phys. F: Met. Phys. 11, 121 (1981).

[11] S. Hüfner, F. Schumann, E. Rotenberg, J. Tobin, S.-H. Yang, B. S. Mun, S. Morton, J. Schäfer, and D. Ehm, Phys. Rev. B 63, 085106 (2001).

[12] Yu. Kucherenko, M. Finken, S. L. Molodtsov, M. Heber, J. Boysen, C. Laubschat, and G. Behr, Phys. Rev. B 65, 165119 (2002).

[13] See Supplemental Material at http://link.aps.org/supplemental/ 10.1103/PhysRevB.92.180407 for additional data concerning the LRE-TM samples such as magneto optical spectra, in-plane hysteresis loops, VSM measurements at different temperatures, TR-MOKE measurements at different fluences and out-of-plane MOKE loops for two HRE-TM samples.

[14] R. C. Taylor, T. R. McGuire, J. M. D. Coey, and A. Gangulee, J. Appl. Phys. 49, 2885 (1978).

[15] R. C. Taylor and A. Gangulee, Phys. Rev. B 22, 1320 (1980).

[16] J. M. D. Coey, D. Givord, A. Lienard, and J. P. Rebouillat, J. Phys. F: Met. Phys. 11, 2707 (1981).

[17] D. Ryan, L. Liao, and Z. Altounian, Solid State Commun. 66, 339 (1988)

[18] P. Hansen, C. Clausen, G. Much, M. Rosenkranz, and K. Witter, J. Appl. Phys. 66, 756 (1989).

[19] P. Hansen, D. Raasch, and D. Mergel, J. Appl. Phys. 75, 5267 (1994).

[20] J. Kübler, Phys. Lett. A 81, 81 (1981).

[21] U. Krey, U. Krauss, and S. Krompiewski, J. Magn. Magn. Mater. 103, 37 (1992).

[22] R. Lorenz and J. Hafner, J. Magn. Magn. Mater. 140-144, 257 (1995).

[23] R. Harris, M. Plischke, and M. Zuckermann, Phys. Rev. Lett. 31, 160 (1973).

[24] R. Cochrane, R. Harris, and M. Plischke, J. Non-Cryst. Solids 15, 239 (1974).

[25] J. A. Gerber, D. J. Miller, and D. J. Sellmyer, J. Appl. Phys. 49, 1699 (1978).

[26] E. Callen, Y. J. Liu, and J. R. Cullen, Phys. Rev. B 16, 263 (1977). 